\title{
Redaktionelle Vorbemerkung: Das „Forum“ als Ort für Meinungsbeiträge
}

\author{
K. Beck $\cdot$ C. Eilders $\cdot$ I. Engelmann $\cdot$ C. Pentzold $\cdot$ Gunter Reus
}

Angenommen: 3. Februar 2021 / Online publiziert: 11. Februar 2021

(C) Der/die Autor(en) 2021

Der folgende Beitrag im „Forum“, der vorab online zugänglich war, hat bei vielen Leserinnen und Lesern erhebliche Empörung ausgelöst. Die Kritik an den Positionen von Rudolf Stöber und seiner Argumentation kam nicht überraschend, gerade weil er im Kern kommunikationsethische und kommunikationspolitische Fragen berührt. Die Beiträge der Rubrik „Forum“ sollen auch kontroverse Diskurse anstoßen.

Um sicherzustellen, dass zeitnah Widerspruch artikuliert wird, hatten wir von den Sprecherinnen der Fachgruppe Medien, Öffentlichkeit und Geschlecht die Zusage für eine Replik eingeholt. Diese ist nun zurückgezogen worden mit der Begründung, der Beitrag sei nicht geeignet, eine Debatte zu eröffnen. Die Absage verweist auf den polemischen Ton des Beitrags, der als diffamierend wahrgenommen wird, u. a. mit seinen Hinweisen auf den Nationalsozialismus und andere autoritäre Regime. Die Kritik ist in einem offenen Brief (http://dgpuk-genderstern.de/) nachzulesen. Diese Entgegnungen machen deutlich, dass der Beitrag unsere Erwartungen an das „Forum“, nicht eingelöst hat, und wir bedauern sehr, dass Teile des Diskurses nun andernorts stattfinden. Wir hoffen, dass die verschiedenen Austragungsorte der Kontroverse zu einem späteren Zeitpunkt noch integriert werden können. Wir erwarten eine längere Debatte und sind sehr froh, dass verschiedene Kolleginnen und Kollegen Beiträge für das nächste Heft der Publizistik zugesagt bzw. bereits formuliert haben.

Wir entnehmen den Entgegnungen, die uns erreicht, und den Gesprächen, die wir geführt haben, dass die Empörung über den Beitrag zum Teil darauf gründet, dass undeutlich ist, für welche Formen der Meinungsäußerung die Rubrik „Forum “ offen sein soll und wo die Grenzen liegen. Einen solchen Diskursraum zu ermöglichen ist für eine Fachzeitschrift vielleicht nicht typisch. Allerdings begreifen wir das „Forum “ als besonderes Profilmerkmal der Publizistik, wobei es auch eine Herausforderung

Prof. Dr. K. Beck ( $\bowtie)$

Universität Greifswald, Greifswald, Deutschland

E-Mail: klaus.beck@uni-greifswald.de 
für die herausgeberische Arbeit bedeutet, mit den eingehenden Beiträgen und Repliken angemessen umzugehen. Die Publizistik will der Community einen Raum für Diskurse zur Verfügung stellen, der lange wenig genutzt wurde, aber seit der Debatte um die Öffnung der Kommunikationswissenschaft für Theorien und Methoden zur Digitalisierung wieder auf Interesse gestoßen ist. Wir verstehen den offenen Brief auch als Ausdruck der unabdingbaren kritischen Begleitung dieser Anstrengungen durch die Fachgemeinschaft.

Übrigens ist der umstrittene Beitrag, wie in den letzten Monaten üblich, im Open Access unter dem DEAL-Vertrag erschienen und nicht aus den DGPuK-Abonnements finanziert.

Einige, die den Diskursraum im Grundsatz befürworten, waren irritiert über den unsachlichen, gar polemischen Stil des Beitrags, zumal diese Form der Stellungnahme in einer wissenschaftlichen Fachzeitschrift nicht erwartet wurde. Wir sind der Ansicht, dass Meinungsaustausch im Sinne der Kommunikationsfreiheit auch durch Provokation und Überspitzung angeregt werden kann, dass er sich darin jedoch nicht erschöpfen sollte. Die Repliken sollen ein Ort sein, wo die Regeln des Diskurses ausgehandelt werden. Hier dürfen nicht nur Argumente und Beweise eingefordert werden, sondern das „Forum“ bedingt Respekt und Verständigungsorientierung. Die Grenze verläuft selbstverständlich dort, wo Menschen diffamiert und Rechte verletzt werden. Diese Grenze hat Rudolf Stöber aus unserer Sicht in dem Beitrag nicht überschritten. Ob andere das auch so sehen, wird sicher in den nächsten Beiträgen deutlich werden. Wir laden dazu ein, im „Forum “ zu diskutieren, inwiefern wissenschaftliche Fachzeitschriften neben der Veröffentlichung von Forschungsaufsätzen mit Peer review, Berichten aus dem Fach und Buchrezensionen auch einen Ort für Meinungsaustausch betreiben sollten und welchen Grundsätzen dieser folgen muss. Bei diesen wichtigen Fragen sollte auch das inhaltliche Anliegen nicht aus dem Blick geraten: Was kann die Kommunikationswissenschaft an fachlichen Argumenten zur öffentlichen Debatte über Fragen der Geschlechtergerechtigkeit beitragen? Auch diese Diskussion möchten wir gerne weiter führen und laden daher zu Debattenbeiträgen, gerne aber auch zu wissenschaftlichen Forschungsartikeln ein.

Funding Open Access funding enabled and organized by Projekt DEAL.

Open Access Dieser Artikel wird unter der Creative Commons Namensnennung 4.0 International Lizenz veröffentlicht, welche die Nutzung, Vervielfältigung, Bearbeitung, Verbreitung und Wiedergabe in jeglichem Medium und Format erlaubt, sofern Sie den/die ursprünglichen Autor(en) und die Quelle ordnungsgemäß nennen, einen Link zur Creative Commons Lizenz beifügen und angeben, ob Änderungen vorgenommen wurden.

Die in diesem Artikel enthaltenen Bilder und sonstiges Drittmaterial unterliegen ebenfalls der genannten Creative Commons Lizenz, sofern sich aus der Abbildungslegende nichts anderes ergibt. Sofern das betreffende Material nicht unter der genannten Creative Commons Lizenz steht und die betreffende Handlung nicht nach gesetzlichen Vorschriften erlaubt ist, ist für die oben aufgeführten Weiterverwendungen des Materials die Einwilligung des jeweiligen Rechteinhabers einzuholen.

Weitere Details zur Lizenz entnehmen Sie bitte der Lizenzinformation auf http://creativecommons.org/ licenses/by/4.0/deed.de. 